\title{
ANÁLISE DE VELOCIDADE MÉDIA E RITMO EM UM DESAFIO DE CORRIDA DE RUA DURANTE A QUARENTENA
}

DOI: 10.48140/digitaleditora.2020.002.14

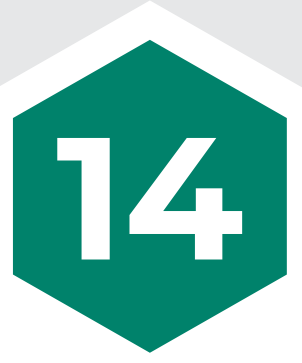

\section{RESUMO}

Objetivos: Nesse contexto, nosso estudo teve como objetivo analisar a velocidade média e ritmo desenvolvido em um desafio de corrida de rua durante a quarentena.

Métodos: Participaram desse estudo 19 corredores sendo 11 do sexo feminino como também 8 do gênero masculino, assim foram analisadas variáveis do ritmo médio como velocidade média dos participantes.

Resultados: Os resultados obtidos revelaram que houve uma diferença para às duas variáveis estudadas quando comparados homens e mulheres, visto que o público masculino obteve os melhores benefícios.

Conclusão: Contudo, no presente estudo conclui-se que o gênero masculino apresentou resultados superiores no ritmo médio como na velocidade média quando comparado ao feminino.
Diessica Lima Araújo

Graduanda em Educação Física da Faculdade AESPI - Ensino Superior do Piauí - Teresina - Piauí

iD

https://orcid.org/00000002-1003-682X

Nayara Rosa Santos Torres

Graduanda em Educação Física da Faculdade AESPI - Ensino Superior do Piauí

Teresina - Piauí

iD

https://orcid.org/0000 0002-0511-9049

\section{Tâmyack Alves de Macêdo}

Profissional de Educação Física, Especialista e Professor Auxiliar da Faculdade AESPI - Ensino Superior do Piauí- Teresina - Piauí 


\section{ANALYSIS OF AVERAGE SPEED AND PACE \\ IN A STREET RUNNING CHALLENGE \\ DURING QUARANTINE \\ DOI: 10.48140/digitaleditora.2020.002.14}

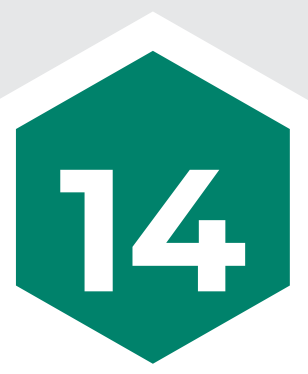

\section{ABSTRACT}

Recebido em: 10/12/2020

Aprovado em: 28/12/2020

Conflito de Interesse: não

Suporte Financeiro: não houve
Objectives: In this context, our study aimed to analyze the average speed and pace developed in a street running challenge during quarantine.

Methods: 19 runners participated in this study, 11 of whom were female as well as 8 men, so variables of the average pace were analyzed, as well as the participants' average speed.

Results: The results obtained revealed that there was a difference for the two variables studied when comparing men and women, since the male audience obtained the best benefits.

Conclusion: However, in the present study it is concluded that the male gender presented superior results in the average rhythm as in the average speed when compared to the feminine. 


\section{(8) \\ INTRODUÇÃO}

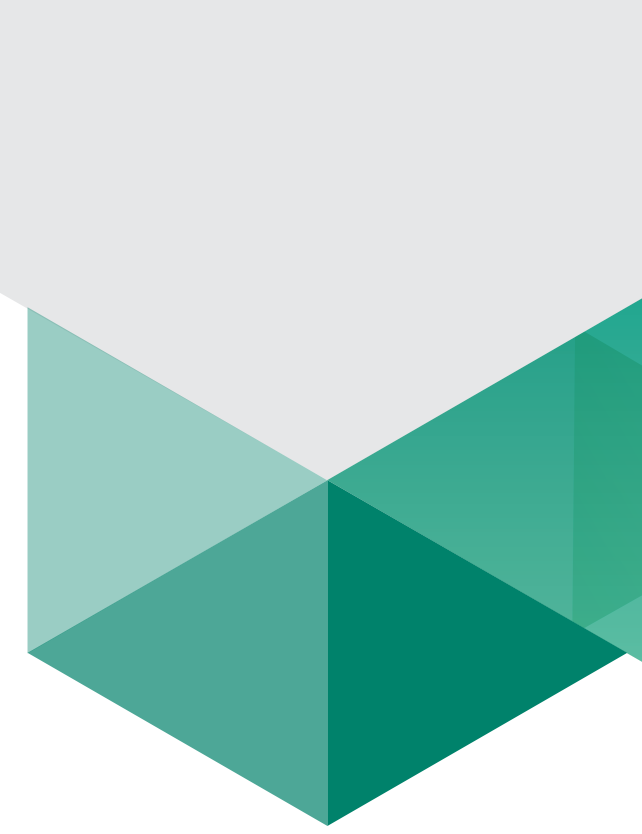

A corrida é compreendida como uma forma de locomoção altamente complexa que requer acentuada coordenação de movimentos. Corredores são todos aqueles que correm em locais abertos, com uma frequência de três vezes por semana e que participam ou buscam participar de competições (EUCLIDES; BARROS; COÊLHO, 2016). Ademais, as corridas de rua se enquadram no conjunto de provas que formam a modalidade do atletismo, e no que the concerne é regulamentada e regida pelas instituições representantes deste esporte, que no Brasil fica a cargo da Confederação Brasileira de Atletismo (CBAt) e internacionalmente pela Associação Internacional de Federações de ginástica (IAAF) (ROJO, 2014).

Há 10 anos, o atletismo era visto como esporte praticado, em sua maioria, por atletas profissionais. No entanto, com a profissionalização das corridas de rua e o surgimento das assessorias esportivas, a corrida vem atraindo, cada vez mais, aquelas pessoas que não se interessam pela profissionalização (TRUCCOLO; MADURO; FEIJÓ, 2008).

Segundo Salgado e Chacon-mikahil (2006) a demanda pela prática da corrida de rua ocorre por inúmeros interesses, que abrange desde a promoção da saúde, integração social como a busca por atividades prazerosas ou competitivas.

Em detrimento dessa questão o crescimento dos praticantes de corrida de rua pode ser observado pelo aumento do número de provas, causado, principalmente, pelo maior envolvimento de praticantes recreacionais. Considerando a dedicação destes praticantes ao treinamento e a busca dos melhores resultados com base em suas potencialidades, parece interessante, mesmo para esse grupo de indivíduos, a identificação dos fatores determinantes do desempenho e suas variáveis relacionadas com o intuito de otimizar os tempos dedicados ao treinamento (SANTOS et al., 2012).

De acordo com Manoel et al. (2015), A utilização de estratégias de ritmo de corrida é decisiva para o desempenho e sucesso dos corredores assim sendo definidas como o conjunto de ajustes de velocidade na tentativa de percorrer no menor tempo possível uma distância predeterminada. Sendo assim, essas estratégias são fundamentais para obtenção de mais velocidade como um melhor ritmo de corrida. Adicionalmente o ritmo em eventos esportivos de longa distância, como ciclismo, corrida ou triatlo podem ser definidos como a distribuição eficiente de reservas energéticas, potência e velocidade ao longo de todo percurso, sem uma desaceleração significativa. Para um ritmo de longa distância bem-sucedido, é necessário selecionar uma estratégia de ritmo adequada (CUK et al., 2019). 
Congruente a isso estudo de Nikolaidis, Cuk, Knechtle (2019), relatam que o ritmo de corrida vem sendo bastante estudado em esportes de resistência e ultra resistência, além de alegar que durante a maratona os atletas tendem a adotar um ritmo positivo, ou seja, sua velocidade diminuiu ao longo da corrida, acompanhada pela presença de um estirão no momento final. Atrelado a isso, esses autores também enunciam que esse mesmo ritmo é utilizado em distâncias mais curtas como 800 M, 10 km e $5 \mathrm{~km}$.

No entanto, com a atual situação que nos encontramos em 2020, devido à pandemia do covid-19, todos tiveram que reajustar suas rotinas passando a viver com distanciamento social.

Notoriamente, um período de reclusão domiciliar à população tende a estimular uma rotina sedentária, o que favorece a um aumento no ganho de peso corporal e surgimento de comorbidades associadas a maior risco cardiovascular, como obesidade, aumento da pressão arterial, intolerância à glicose, bem como transtornos psicossociais como ansiedade e depressão (FERREIRA et al., 2020). Frente a isso a prática de atividade física de forma sustentável que seguissem as indicações da Organização Mundial da Saúde (OMS) foram um dos grandes desafios durante um período tempestuoso, a prática de corrida de forma isolada longe de aglomerações e buscando locais isolados foi uma das soluções encontradas para realizar atividade física.

Desse modo, o objetivo desse estudo foi analisar a velocidade média e ritmo em um desafio de corrida de rua durante a quarentena em competidores amadores e profissionais.

\section{METODOLOGIA}

A O presente estudo trata-se de uma análise documental dos registros de resultados "Desafio Prorunner etapa bronze", essa atividade foi proposta pela assessoria esportiva "PRORUNNER", os resultados foram obtidos por meio do aplicativo software STRAVA versão 179.0.0.0. A corrida de rua ocorreu no município de Teresina - Piauí. Foi realizado o estudo no período da pandemia do (COVID-19) entre os dias 20 a 29 de julho de 2020, do qual foi feito um desafio de corrida de rua, tanto para corredores amadores como para profissionais, dentre eles maratonistas e ultra maratonistas.

O desafio tinha como principal objetivo fazer com que os atletas realizassem a corrida durante os 9 dias consecutivos, podendo o participante correr ou mesmo fazer caminhada. Os $5 \mathrm{~km}$ (quilômetros) era o objetivo por dia, mas ficava a critério do atleta a divisão ou estratégia que melhor se adequasse. Uma das regras principais era concluir durante os 9 dias um total de $45 \mathrm{~km}$ (quilômetro) no final, sendo obrigatória a participação em todos os dias para não serem desclassificados, a segunda regra estabelecida foi que os atletas tinham até 24 horas para validação de seus respectivos treinos e como terceira regra a validação dos treinos teria que ser através do aplicativo STAVA no qual seria gerenciado pela organização do evento.

Participaram do desafio 84 indivíduos, sendo excluídas 65 por não completarem os 9 dias consecutivos da competição. Portanto, 
foram coletados os dados de 19 corredores sendo 11 do sexo feminino como também 8 do gênero masculino. Os dados foram coletados através do aplicativo STRAVA. Além disso, o local da realização da corrida ficou a critério de cada competidor.

Os dados foram organizados em planilhas no software Excel contendo as seguintes mensurações dos competidores: horário do treino, distância percorrida, ritmo médio, ganho de elevação. Contudo, foram isoladas algumas variáveis para análises como: velocidade média e ritmo dos participantes, esses dos quais foram separados por gênero.

\section{RESULTADOS E DISCUSSÃO}

Os dados analisados nesse estudo mostraram que houve uma diferença em relação ao ritmo médio dos atletas masculino e feminino, como pode ser observado no gráfico abaixo.

GRÁFICO 1. Ritmo médio feminino e masculino durante um desafio de corrida de rua.

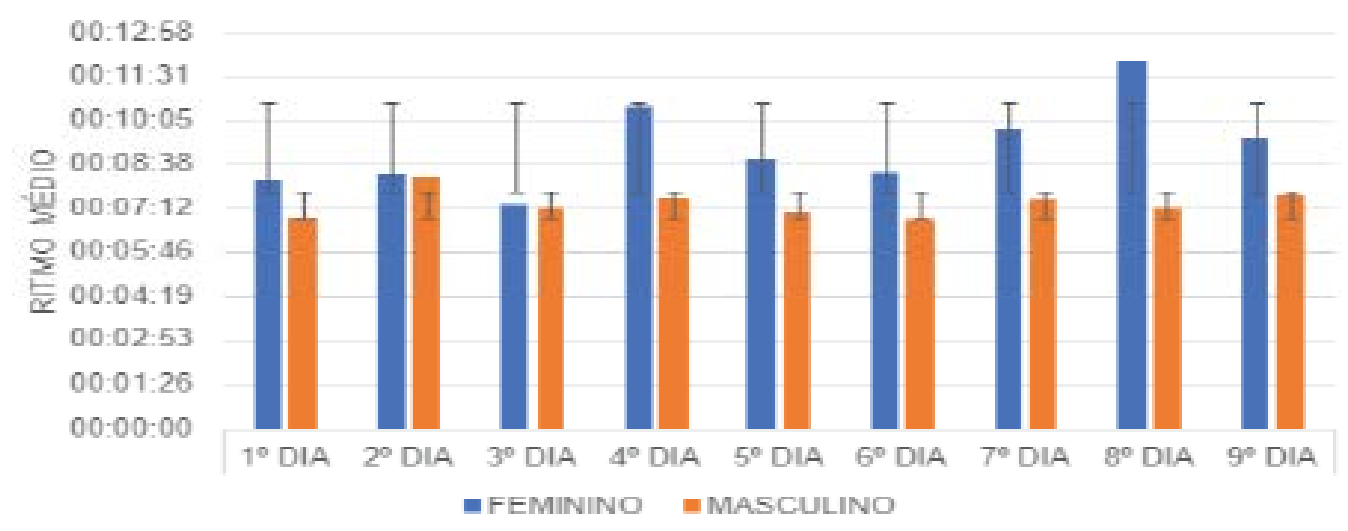

Fonte: Dados da pesquisa.

Em contrapartida, o estudo de Breen et al. (2018) mostrou que as mulheres apresentaram um ritmo significativamente menor quando comparado aos homens (diferença média $=-3,81 \%, P<0,001$ ).

Resultados semelhantes ocorreram em estudo de Deaner e Lowen (2016), do qual homens correram $21-22 \%$ mais rápido do que as mulheres. Essas das quais diminuíram mais a velocidade do que os homens $(p=0,002)$. No entanto, essa diferença de sexo no ritmo era comum em magnitude $(E S=$ $0,10)$.

Segundo Ramalho, Nicolau e Toledo (2009) movimento progressivo é quando o móvel caminha a favor da orientação positiva da trajetória, ou seja, quando há um deslocamento no sentido de distanciamento do ponto inicial, com base no conceito de movimento progressivo, temos o conceito de velocidade média que a relação entre a distância percorrida e o tempo gasto para a realização da mesma, sendo as principais unidades de medidas utilizadas $\mathrm{m} / \mathrm{s}$ ou $\mathrm{km} / \mathrm{h}$, com isso também apresentou diferença quando comparado homens e mulheres como pode ser observado no gráfico 2 . 
GRÁFICO 1. Velocidade média feminina e masculino durante um desafio de corrida de rua.

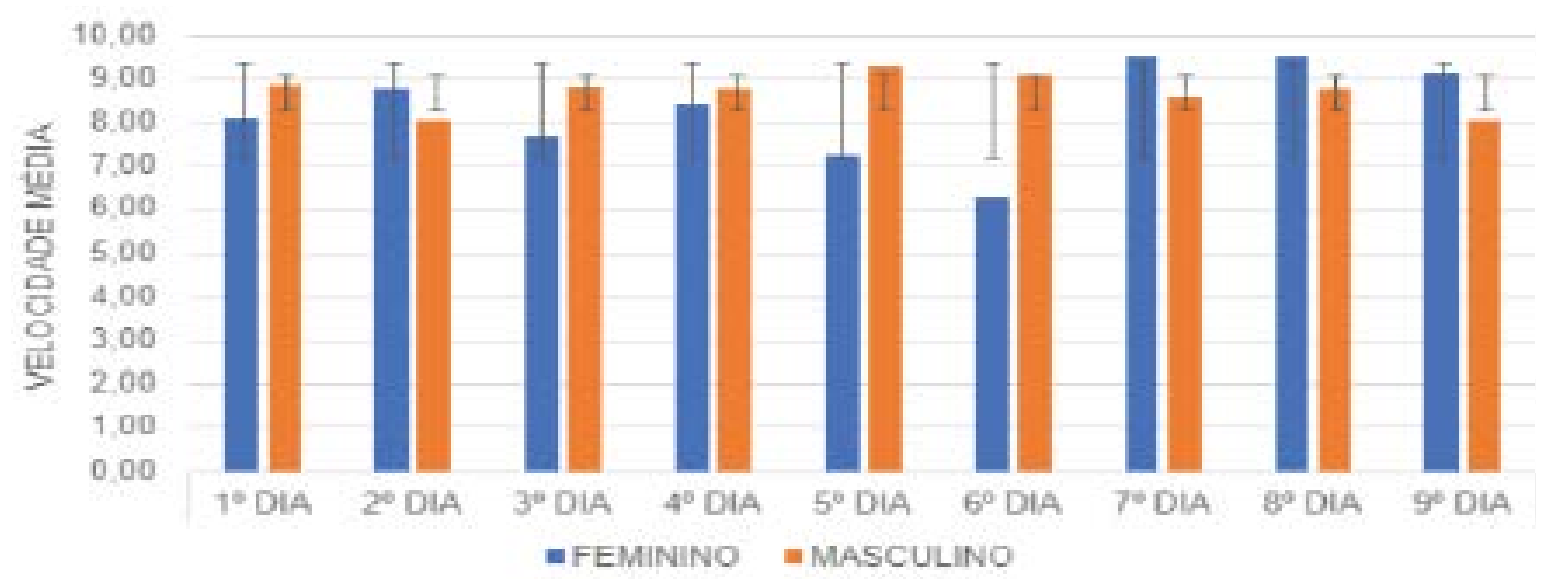

Fonte: Dados da pesquisa.

Como pôde ser observado com o gráfico acima nota-se que em relação a essa variável houve diferença entre os gêneros. Entretanto, o grupo masculino mesmo com uma menor quantidade de participantes, esses ainda conseguiram obter melhores resultados. Similarmente, para Cuk et al. (2019), foi mostrada uma associação de sexo x raça, ( $\chi 2=293,6, p<0,01, \phi=0,13)$, onde a proporção de homens para mulheres foi menor na meia maratona $(1,99)$ em comparação com a maratona $(3,77)$. Como também foi observada uma diminuição gradual na velocidade média ao longo dos segmentos de corrida para ambos os sexos.

Em comparação, estudos de Nikolaidis, Cuk, Knechtle (2019) que avaliaram homens e mulheres em meia maratona e maratona mostraram que houve uma diferença na velocidade média entre ambos de apenas $0,030 \%(p=0,67)$.

Torres (2017) elucidaram em seus resultados que a velocidade média dos treinos de corrida, houve uma frequência relativa de (41,5\%) nos homens, do qual ocorreram no ritmo $>10 \mathrm{a} 12 \mathrm{~km} / \mathrm{h}$, enquanto o maior grupo das mulheres, num percentual próximo $(38,0 \%)$, corria numa agilidade $>8$ a $10 \mathrm{~km} / \mathrm{h}$. Como pode ser observado o grupo masculino percorria uma maior quilometragem média e máxima nos treinamentos realizados, em relação às mulheres. Em comparação ao nosso estudo Torres (2017) também relatou que os homens treinaram menos dias por semana, levando-se em conta a realização de qualquer treinamento, mas, em sua maioria, faziam quantidade similar de sessões semanais (28,4\% e 39,3\%, nas faixas de $3-4$ e $5-6$ treinos/semana, respectivamente), comparativamente às mulheres $(27,1 \%$ e $39,8 \%$, nas mesmas faixas). Isto pode sugerir que, no masculino, os praticantes estavam concentrando mais sessões em menos dias de treinamento. Fato semelhante aos observados em nosso estudo. 
Vale ressaltar que houve dificuldade de encontrar achados sobre essas variáveis estudadas principalmente em corridas de curta duração, desse modo são necessários estudos mais aprofundados sobre essas variáveis.

Foram inscritos 84 participantes no desafio de corrida, que tinha como objetivo fazer um determinado número de dias seguidos a quantidade de $5 \mathrm{~km}$, sendo que esta quilometragem poderia ser feita tanto na forma de caminhada, corrida ou mescla dos dois tipos, um dos critérios de desclassificação era deixar de efetuar a atividade por pelo menos um dia da competição, valendo ressaltar que não era obrigatório executar $5 \mathrm{~km}$ todos os dias, mas ao final da disputa teria que em média ter sido obtido esse resultado, com isso apenas $22,62 \%$ dos registrados concluíram a disputa, dos $77,38 \%$ que não finalizaram o mesmo, 5,95\% não o fizeram porque contraíram a COVID-19, 11,90\% tiveram que parar por conta de dores nos joelhos devidos à atividade serem em dias consecutivos, 3,57\% desistiram sem justificar o motivo, 55,95\% foram desclassificados por não terem inserido os dados no aplicativo que estava registrando o conhecimento de todos os competidores. 


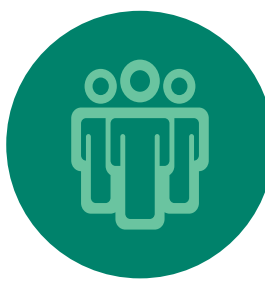

\section{CONCLUSÃO}

O presente estudo conclui-se que o gênero masculino apresentou melhores resultados no ritmo médio como na velocidade média quando comparado ao feminino. Dentre dos quais os fatores biológicos, gênero, período do dia e o ambiente físico fizeram-se relevantes para análise do ritmo como para a velocidade média no desenvolver da competição e com isso os resultados obtidos, alguns participantes corriam apenas nas primeiras horas da manhã enquanto outros no final do dia ou parte da noite, com isso todo o desgaste do dia de trabalho, influência no rendimento do treino.

Apesar de todos os diversos fatores influenciadores, o objetivo da competição foi concluído com êxito pelos participantes que buscaram a superação diária, pois a prática de atividade em 9 dias consecutivos acarreta adaptações fisiológicas, que se tornam benéficas a longo prazo, pois apesar desses corredores sofrer um desgaste agudo a princípio esse se torna favorável ao nível crônico. 


\section{REFERÊNCIAS}

BREEN, D. et al. Marathon Pace Control in Masters Athletes. International Journal of Sports Physiology and Performance, v. 13, n. 3, 2018.

CUK, I. et al. Age Differences in Pacing in Endurance Running: Comparison between Marathon and Half-Marathon Men and Women. Medicina, 2019, 55, 479.

DEANER, R. O.; LOWEN, A. Males and females pace differently in high school cross country races. Journal of Strength and Conditioning Research, 2016; 30(11): 2991- 2997.

EUCLIDES, M. F.; BARROS, C. L; COÊLHO, J. C. A. Benefícios da corrida de rua. Revista Conexão Eletrônica, Três Lagoas, v. 13, n. 1, 2016.

FERREIRA, M.J. et al. Vida Fisicamente Ativa como Medida de Enfrentamento ao COVID-19. Arquivos Brasileiro de Cardiologia, 2020; 114(4):601-602.

MANOEL, F. A. et al. Influência do nível de performance na estratégia de ritmo de corrida em prova de 10 km de corredores recreacionais. Revista Brasileira de Educação Física e Esporte, 2015, jul./Set; 29(3):355-60.

MOURA, C. A. et al. Características associadas aos corredores da maratona do rio de janeiro. Revista Fitness e Performance Journal, 2010, jan./mar; 9(1):106-112.

NIKOLAIDIS, P. T.; CUK, I; KNECHTLE, B. Pacing of Women and Men in Half-Marathon and Marathon Races. Medicina, 2019, 55, 14.

RAMALHO; NICOLAU; TOLEDO, Os fundamentos da Física, 2009, 10ae edição, pág 34-35.

ROJO, J. R.. Corridas de rua, sua história e transformações. In: VII CONGRESSO SULBRASILEIRO DE CIÊNCIAS DO ESPORTE, 2014, Matinhos. Anais do Congresso Sulbrasileiro de Ciências do Esporte, 2014, Disponível em: http://cbce.tempsite.ws/congressos/index.php/7csbce/2014/index.

SALGADO, J. V. V.; CHACON-MIKAHIL, M. P. T. Corrida de rua: análise do crescimento do número de provas e de praticantes. CONEXÕES, Campinas, v. 4, n. 1, p. 90-99, 2006.

SANTOS, T. M. et al. vo2 máx estimado e sua velocidade correspondente predizem o desempenho de corredores amadores. Revista Brasileira de Cineantropometria e Desempenho Humano, 2012, 14(2):192-201.

TORRES, F. C. Associação entre variáveis demográficas, antropométricas e de treinamento com o desempenho em corredores de rua recreacionais. Dissertação (Mestrado)- Universidade Federal do Paraná. Setor de Ciências Biológicas. Programa de Pós-Graduação em Educação Física. Curitiba, 79 f. 2017.

TRUCCOLO, A. B.; MADURO, P. A; FEIJÓ, E. A. Fatores motivacionais de adesão a grupos de corrida. Motriz, Rio Claro, v.14 n. 2 p. 108-114, abr./jun. 2008. 